\title{
Modelización mecánica del enfriamiento rápido en sistemas tipo gres porcelánico
}

\author{
M. DAL BÓ(1) , V. CANTAVELLA ${ }^{(2)}$, E. SÁNCHEZ ${ }^{(2)}$, D. HOTZA ${ }^{(1)}$, A. BOSCHI ${ }^{(3)}$ \\ (1) Universidade Federal de Santa Catarina (UFSC). Brasil \\ (2) Instituto de Tecnología Cerámica. Asociación de Investigación de las Industrias Cerámicas. Universitat Jaume I. Castellón, España \\ (3) Universidade Federal de São Carlos (UFSCar). Brasil
}

Este trabajo ha sido presentado como comunicación oral, tras su evaluación por el Comité Científico, en el XII Foro Global del Recubrimiento Cerámico. QUALICER (13 y 14 febrero 2012. Castellón. España).

\begin{abstract}
En este trabajo se analiza el efecto del enfriamiento sobre el comportamiento mecánico y, en particular, sobre las tensiones residuales que aparecen cuando se trabaja con materiales tipo gres porcelánico. Estas composiciones tienen una microestructura muy compleja, donde se encuentran varias fases cristalinas y la fase vítrea dista de ser homogénea. Por ello se planteó trabajar con una composición más sencilla, empleando como material de partida feldespato sódico al que se añadió cuarzo de diferente tamaño de partícula.

Se utilizó un modelo viscoelástico para la estimación de las tensiones residuales que se producen durante el enfriamiento. Los parámetros del modelo se obtuvieron de la bibliografía o se determinaron en ensayos de laboratorio.

Se diseñó un montaje que permitía la medida sin contacto de la temperatura en las superficies superior e inferior de la pieza durante el enfriamiento. Las probetas fueron sometidas a diferentes tipos de enfriamientos y, a continuación, se determinaron las tensiones residuales mediante el método de relajación de las deformaciones por corte.
\end{abstract}

Palabras clave: tensiones residuales, viscoelasticidad, modelización, enfriamiento, resistencia mecánica.

Mechanical modelling of rapid cooling in porcelain tile-type systems

This paper analyses the effect of cooling on mechanical behaviour, in particular, on the residual stresses that appear when materials of the porcelain tile type are involved. However, these compositions have a very complex microstructure, in which there are several crystalline phases and the glassy phase is not homogeneous. In this study a simpler composition was therefore formulated, using sodium feldspar as starting material to which quartz with different particle sizes was added.

A viscoelastic model was used to estimate the residual stresses that develop during cooling. The parameters of the model were obtained either from the literature or were determined in laboratory tests.

An assembly was designed that allowed non-contact measurement of the temperature at the top and bottom surfaces of the test pieces during cooling. The test pieces were subjected to different types of cooling and their residual stresses were then determined by the strain relaxation slotting method.

Keywords: residual stresses, viscoelasticity, modelling, cooling, mechanical strength.

\section{INTRODUCCIÓN}

Es bien conocido que, en piezas cerámicas, el enfriamiento rápido conduce a tensiones residuales en las piezas a la salida del horno. Esto sucede incluso con productos que generan poca fase vítrea a elevada temperatura (revestimiento poroso). Las tensiones residuales son importantes porque influyen sobre:

- La resistencia mecánica de las piezas.

- Las curvaturas en diferido, aunque éstas dependen mucho de la fluencia del material.

- El proceso de corte durante la instalación de las baldosas. Las tensiones residuales pueden hacer que las piezas se corten según trayectorias no deseadas, o que se rompan.

- En el rectificado, la eliminación de zonas tensionadas puede provocar un cambio de curvatura.
Además, las tensiones durante el enfriamiento de la pieza en el interior del horno podrían provocar deterioro microestructural o incluso la rotura de la pieza.

Existen varios tipos de tensiones residuales, aunque este trabajo se centra solo en uno de ellos: el asociado a los gradientes térmicos dentro de la pieza durante el enfriamiento.

\section{MODELO TEÓRICO}

El modelo teórico (1) consta de dos etapas (figura 1). En la primera se resuelve el problema térmico, consistente en calcular el perfil de temperatura; para ello se necesita conocer la geometría de la pieza, las propiedades térmicas (conductividad térmica y calor específico), la densidad y la temperatura de la superficie en función del tiempo. 
La ecuación de transmisión de calor en estado no estacionario adopta la forma (2):

$$
\text { Ec. } 1 \frac{\partial \mathrm{T}}{\partial \mathrm{t}}=\alpha \nabla^{2} \mathrm{~T}
$$

donde:

$\mathrm{T}$ : temperatura en un punto de la pieza y en un instante dado $(\mathrm{K})$

$\nabla^{2}$ : operador laplaciano $\left(\mathrm{m}^{-2}\right)$

$\mathrm{t}: \quad$ tiempo (s)

$\alpha$ : difusividad térmica $\left(\mathrm{m}^{2} / \mathrm{s}\right)$

La difusividad térmica $\alpha$ es el cociente entre la conductividad térmica $\mathrm{k}(\mathrm{W} /(\mathrm{m} \cdot \mathrm{K}))$, la densidad $\rho\left(\mathrm{kg} / \mathrm{m}^{3}\right)$ y el calor específico $c_{\mathrm{p}}(\mathrm{J} /(\mathrm{kg} \cdot \mathrm{K})): \alpha=\mathrm{k} /\left(\rho \mathrm{c}_{\mathrm{p}}\right)$.

En la segunda parte se aborda el problema mecánico, determinando los desplazamientos de cada punto de la pieza a partir de las propiedades mecánicas (ley de comportamiento), curva dilatométrica, ecuaciones de equilibrio y perfil térmico.

Las ecuaciones de equilibrio mecánico derivan de los balances de fuerzas y momentos aplicados a la pieza (3), y no dependen del tipo de material ni de sus propiedades. La ley de comportamiento establece la relación entre la tensión y la deformación que sufre un cuerpo y es función del tipo de material. La ley más sencilla es la ecuación de Hooke (elasticidad lineal); sin embargo, esta ley es incapaz de explicar la generación de tensiones residuales. Se requiere una componente no-elástica, por ejemplo la viscoelástica lineal, lo que conduce a la expresión:

$$
\begin{aligned}
\varepsilon_{\mathrm{e}, \mathrm{x}} & =\frac{1}{\mathrm{E}} \sigma_{\mathrm{x}} \\
\text { Ec. } 2 \quad \frac{\mathrm{d} \varepsilon_{\mathrm{v}, \mathrm{x}}}{\mathrm{dt}} & =\frac{1}{3 \eta} \sigma_{\mathrm{x}}
\end{aligned}
$$

donde:

$\varepsilon_{e, x}:$ deformación elástica a lo largo del eje $\mathrm{x}$

$\varepsilon_{\mathrm{v}, \mathrm{x}}:$ deformación viscosa a lo largo del eje $\mathrm{x}$

$\sigma_{x}:$ tensión normal sobre un plano perpendicular al eje $x(\mathrm{~Pa})$

E: módulo de elasticidad $(\mathrm{Pa})$

$\eta$ : viscosidad (Pa.s)

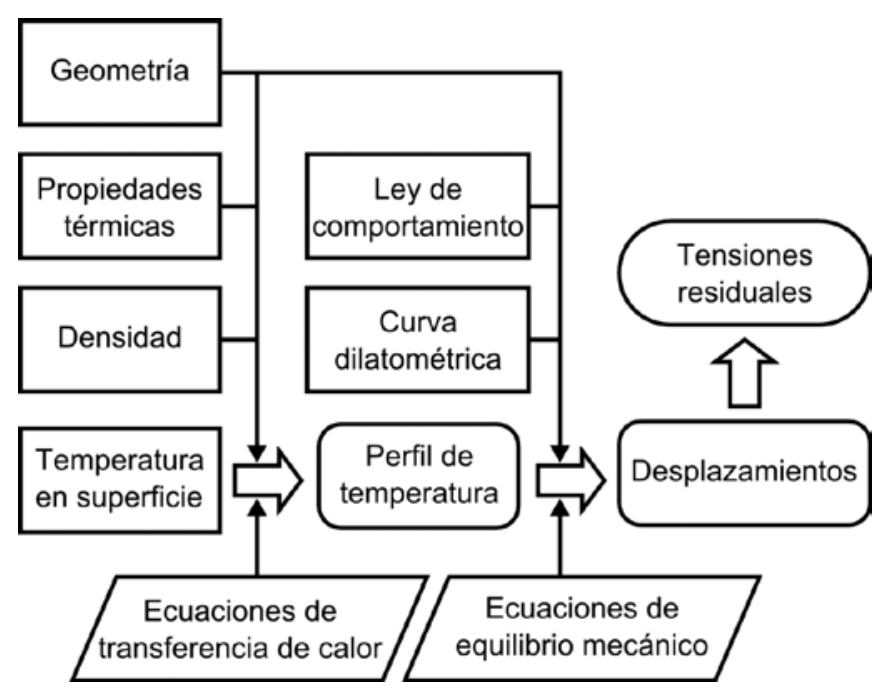

Figura 1. Etapas del cálculo de las tensiones residuales.

Existen varias ecuaciones que relacionan la viscosidad con la temperatura, siendo una de las más sencillas (4):

$$
\text { Ec. } 3 \eta=\eta_{0} \mathrm{e}^{\Theta / \mathrm{T}}
$$

Donde $\eta_{0}$ es el factor pre-exponencial (Pa.s) y $\Theta$ una constante (K).

La resolución de los problemas térmico y mecánico requiere utilizar métodos numéricos. Para el primero de ellos se emplearon diferencias finitas y para el segundo el método de los elementos finitos.

\section{EXPERIMENTAL}

\subsection{Preparación de las probetas}

Para preparar las probetas se partió de feldespato sódico (Mario Pilato). Este feldespato fue molturado con agua hasta alcanzar un tamaño medio de partícula de 6,4 $\mu \mathrm{m}$. El material fue secado en lámpara de infrarrojos y posteriormente desaglomerado por molturación vía seca durante 5 minutos, con lo que no se alteró el tamaño medio de partícula. El polvo fue humectado al 8,0\% y prensado a $35 \mathrm{MPa}$.

\begin{tabular}{|c|c|c|}
\hline Composición & Material & $\left.\mathrm{T}_{\mathrm{md}}{ }^{\circ} \mathbf{C}\right)$ \\
\hline FdtoNa & Feldespato sódico & 1200 \\
FdtoNa-QF & Feldespato sódico +20\% cuarzo Sibelco SE-100 & 1205 \\
FdtoNa-QG & Feldespato sódico +20\% cuarzo Sibelco SE-8 & 1205 \\
& & \\
\end{tabular}




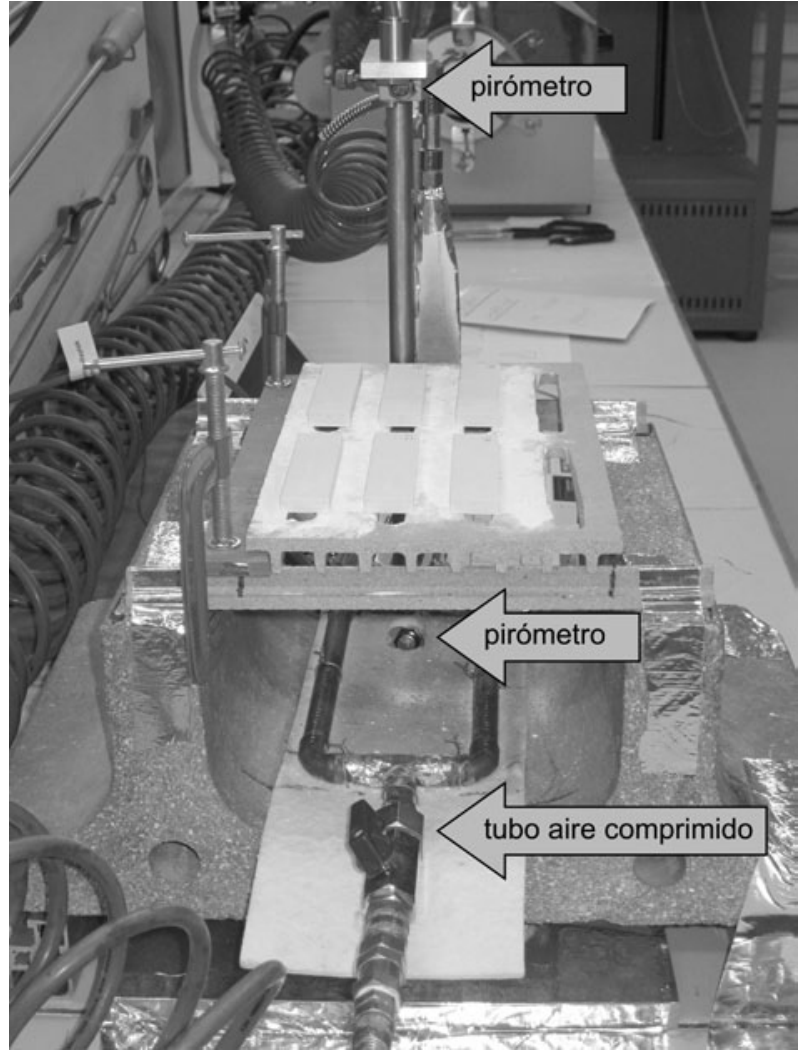

Figura 2. Montaje para la medida de la temperatura durante los enfriamientos.

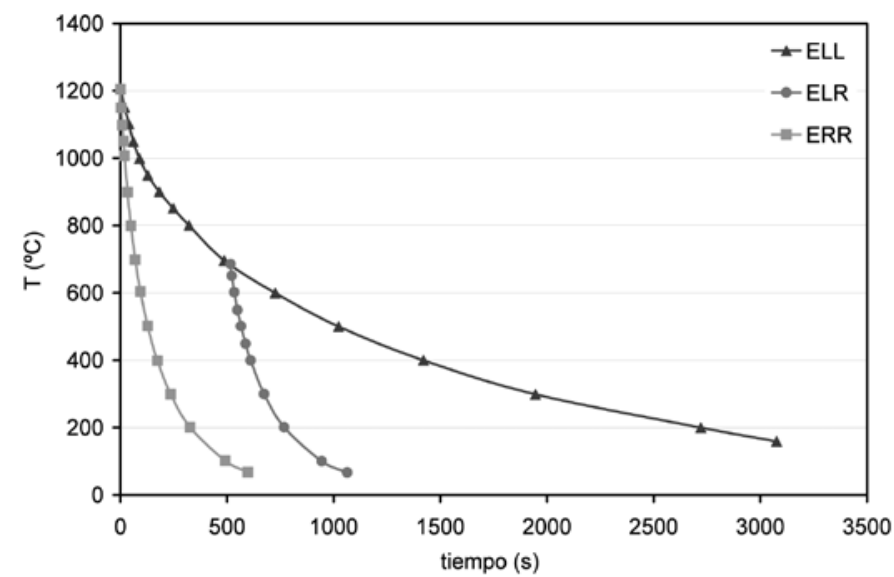

Figura 3. Temperatura de la superficie de las probetas durante los diferentes tipos de enfriamientos.
A partir del feldespato molturado se preparó otro lote de material al que se añadió un $20 \%$ en peso de cuarzo de diferentes granulometrías (Sibelco SE-100 y SE-8, con diámetros medios de 13,4 um y $31 \mu \mathrm{m}$, respectivamente). En la tabla I se muestran los materiales empleados en la elaboración de las probetas. El proceso de conformado de las probetas con cuarzo fue el mismo que el de las probetas de feldespato sódico.

Finalmente las probetas fueron introducidas en estufa a 110 ${ }^{\circ} \mathrm{C}$ durante al menos $2 \mathrm{~h}$, con el fin de eliminar la humedad. A continuación fueron cocidas a la temperatura de máxima densificación (columna $\mathrm{T}_{\mathrm{md}}$ de la tabla I).

Las probetas fueron sometidas a diferentes tipos de enfriamientos, dentro y fuera del horno. En la figura 2 se muestra el dispositivo empleado en los enfriamientos fuera del horno. El montaje consta de un tubo con aire comprimido empleado para enfriar las probetas por su parte inferior, y un par de pirómetros (Raytek modelo MI320LTS) que miden simultáneamente la temperatura de las superficies superior e inferior.

Se realizaron los siguientes tipos de enfriamientos:

- ELL: Enfriamiento lento, en el interior del horno.

- ELR: Enfriamiento lento hasta $650{ }^{\circ} \mathrm{C}$ y rápido (extracción de las probetas del horno y enfriamiento con aire comprimido) desde $650{ }^{\circ} \mathrm{C}$ hasta temperatura ambiente.

- ERR: Enfriamiento rápido desde temperatura máxima hasta temperatura ambiente.

Estos enfriamientos tenían por objetivo separar dos fenómenos que se producen por la elevada velocidad de enfriamiento: la generación de tensiones residuales y el deterioro microestructural. El primero conduce a un incremento en la resistencia mecánica, mientras que el segundo provoca una reducción (5).

En la figura 3 se muestra la evolución de la temperatura media de la superficie de las piezas durante los diferentes tipos de enfriamientos.

\subsection{Medida de las tensiones residuales}

Para medir las tensiones residuales se empleó el método "relajación de la deformación por corte" (Strain Relaxation Slotting Method: SRSM) (6). Este método ha sido empleado en otras ocasiones para medir tensiones residuales en piezas de gres porcelánico $(5,7)$. Consiste en pegar una galga en la parte inferior de la pieza a ensayar y, a continuación, hacer cortes de profundidad creciente $\left(\mathrm{a}_{\mathrm{i}}\right)$ desde la superficie superior, midiendo la deformación registrada por la galga $\left(\varepsilon_{\mathrm{g}}\right)$ (figura 4$)$.
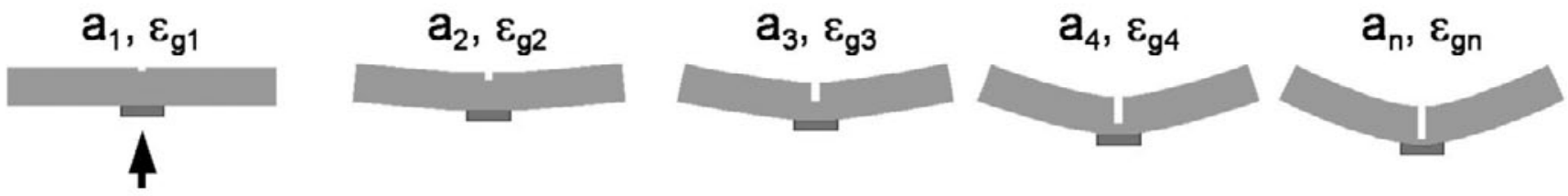

\section{galga extensiométrica}

Figura 4. Representación esquemática del fundamento del método empleado para medir las tensiones residuales. 
Las tensiones residuales en un punto dentro de la pieza $\left(\sigma_{\mathrm{res}}\right)$ dependen de la posición de dicho punto respecto al centro. Esta posición puede cuantificarse mediante la coordenada $\zeta$, siendo $\zeta=0$ el centro, $\zeta=-1$ la superficie inferior y $\zeta=+1$ la superior. La $\sigma_{\text {res }}$ se puede expresar como una combinación lineal de polinomios de Legendre $\left(\mathrm{P}_{\mathrm{k}}\right)$ de la coordenada $\zeta$ (ec. 4). Habitualmente, en piezas cerámicas tipo gres porcelánico y con los enfriamientos convencionales, es suficiente considerar solo el término de segundo grado.

Ec. 4

$\sigma_{\text {res }}=-E \sum_{\mathrm{k}=2}^{\infty} \lambda_{\mathrm{k}} \mathrm{P}_{\mathrm{k}}(\xi) \cong-\mathrm{E} \lambda_{2} \mathrm{P}_{2}(\xi)=\sigma_{\text {sfc }} \mathrm{P}_{2}(\xi)=\sigma_{\text {sfc }} \frac{3 \xi^{2}-1}{2}$

La tensión residual en la superficie es $\sigma_{\mathrm{sfc}}=-\mathrm{E} \lambda_{2} \mathrm{y}$, por tanto, el perfil completo de tensiones residuales se puede expresar solo en función del la tensión en la superficie.

\section{DETERMINACIÓN DE LOS PARÁMETROS DEL MO- DELO}

\subsection{Parámetros térmicos}

Las probetas analizadas están formadas básicamente por cuatro fases: vidrio de albita, cristales de albita, cuarzo y aire (porosidad). La conductividad efectiva de la mezcla puede calcularse utilizando el modelo EMT (Effective Medium Theory) (8-10):

$$
\text { Ec. } 5 \sum_{\mathrm{j}} \mathrm{V}_{\mathrm{j}} \frac{\mathrm{k}_{\mathrm{j}}-\mathrm{k}_{\mathrm{eff}}}{\mathrm{k}_{\mathrm{j}}+2 \mathrm{k}_{\mathrm{eff}}}=0
$$

donde la suma se extiende a todos los componentes, $\mathrm{V}_{\mathrm{j}}$ es la fracción volumétrica de la fase $A_{j}, k_{i}$ es la conductividad térmica de $\mathrm{A}_{\mathrm{j}} \mathrm{y} \mathrm{k}_{\text {eff }}$ la conductividad efectiva. La fracción volumétrica de $\mathrm{A}_{\mathrm{j}}$ se puede calcular a partir de su fracción másica una vez conocida la densidad de cada componente (11).

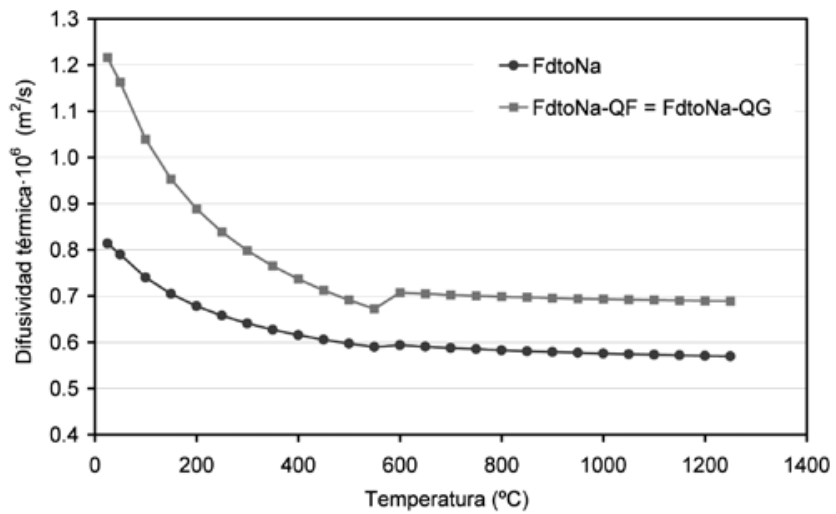

Figura 5. Difusividad térmica de las composiciones utilizadas, calculada empleando la ec. 5 .
Se consideró la variación de todos los parámetros térmicos con la temperatura. De la bibliografía se obtuvo la conductividad térmica de los siguientes componentes: vidrio de albita (12), cristal de albita (12), cuarzo (13) y aire (14). Para determinar la densidad se utilizaron los trabajos de Hofmeister (12) y Ohno (15), y para el calor específico los de Hofmeister (12) y Hemingway (16).

En la figura 5 se muestra la difusividad térmica de las composiciones utilizadas, calculada con el modelo EMT (ec. 5)

\subsection{Parámetros mecánicos}

Los dos parámetros más importantes de la simulación mecánica son la ley de comportamiento y la curva dilatométrica.

Se utilizó una ley de comportamiento viscoelástica lineal, donde se incorporó la variación de la viscosidad con la temperatura. Este modelo tiene dos parámetros: el módulo de elasticidad y la viscosidad.

\subsubsection{MÓDULO DE ELASTICIDAD}

Se determinó experimentalmente el módulo de elasticidad del FdtoNa, FdtoNa-QF y FdtoNa-QG utilizando un equipo de emisión acústica acoplado a un horno (Grindosonic). Con este equipo fue posible medir E hasta la temperatura de transformación $\left(\mathrm{T}_{\mathrm{g}}\right)$ del vidrio de albita.

Por encima de dicha temperatura se utilizaron datos bibliográficos del módulo de elasticidad de cada componente cristalino $(15,17)$, y en el caso del vidrio de albita $\left(E_{a l}\right)$ se empleó la expresión siguiente (18):

Ec. $6 \mathrm{E}_{\mathrm{al}}=\mathrm{E}_{\mathrm{al}, \mathrm{g}} \frac{\mathrm{T}_{\mathrm{al}, \mathrm{g}}}{\mathrm{T}}$

donde $\mathrm{T}_{\mathrm{al}, \mathrm{g}}$ es la temperatura de transición vítrea $(\mathrm{K}), \mathrm{T}$ la temperatura $(\mathrm{K})$ y $\mathrm{E}_{\mathrm{al}, \mathrm{g}}$ el módulo de elasticidad para la temperatura de transición vítrea $(\mathrm{Pa})$. Finalmente se utilizó una ley de mezclas (ec. 7) para calcular el módulo de elasticidad $\left(\mathrm{E}_{\text {eff }}\right)$ de cada probeta:

$$
\text { Ec. } 7 \mathrm{E}_{\mathrm{eff}}=\sum_{\mathrm{j}} \mathrm{V}_{\mathrm{j}} \mathrm{E}_{\mathrm{j}}
$$

Siendo $E_{\mathrm{j}}$ el módulo de elasticidad de cada una de las fases.

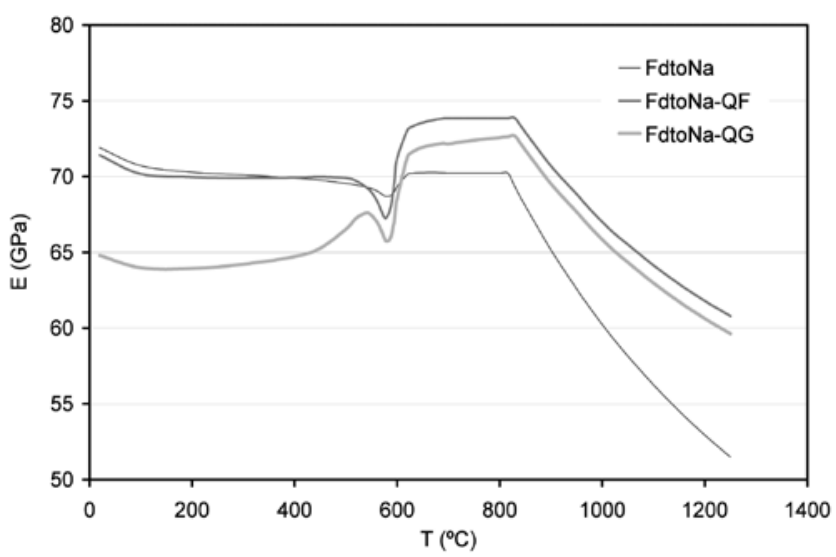

Figura 6. Evolución del módulo de elasticidad con la temperatura. 
TABla II. Temperaturas de transición VÍtrea $\left(\mathrm{T}_{\mathrm{g}}\right)$ y DE SEMIESFERA $\left(\mathrm{T}_{\mathrm{s} 2}\right)$ VISCOSIDAD PARA LAS COMPOSICIONES UtILIZADAS.

\begin{tabular}{|c|c|c|c|c|}
\hline & & \multicolumn{3}{|c|}{ Temperatura $\left({ }^{\circ} \mathrm{C}\right)$} \\
\hline & $\eta($ Pa·s) & FdtoNa & FdtoNa-QF & FdtoNa-QG \\
\hline$T_{\mathrm{g}}$ & $10^{12}$ & 816 & 830 & 830 \\
$T_{\mathrm{s} 2}$ & $10^{3.5}$ & 1465 & 1470 & 1470 \\
\hline
\end{tabular}

\subsubsection{VISCOSIDAD}

La viscosidad del vidrio de albita sigue una ecuación tipo Arrhenius (4) (ec. 3). Esto facilita el cálculo porque es suficiente conocer la viscosidad a dos temperaturas. Se utilizó un dilatómetro (Adamel-Lhomargy, modelo DI-24) para determinar la temperatura de transición vítrea $\mathrm{T}_{\mathrm{g}} \mathrm{y}$ un microscopio de calefacción (Misura HSM, modelo M3M 1600.80.2) para cuantificar la temperatura de semiesfera $T_{\mathrm{s} 2}$ temperaturas a las cuales se asume que la viscosidad es $10^{12}$ $\mathrm{Pa} \cdot \mathrm{s}$ y $10^{3.5} \mathrm{~Pa} \cdot \mathrm{s}$ respectivamente $(19,20)$. En la tabla II se muestran los resultados obtenidos.

\subsubsection{CURVA DILATOMÉTRICA}

La curva dilatométrica se midió utilizando un dilatómetro (Adamel-Lhomargy, modelo DI-24). Estas curvas muestran, a alta temperatura, un inicio de contracción debido a la carga aplicada por el propio palpador del dilatómetro y a la generación de fase vítrea en las probetas. Esta contracción no se produce durante el enfriamiento de la pieza, por lo que en el cálculo se utilizó una extrapolación lineal, como se muestra en la figura 7.

\section{APLICACIÓN DEL MODELO}

En la figura 8 se representa la diferencia de temperatura entre el centro y la superficie de la pieza FdtoNa sometida a diferentes tipos de enfriamientos.

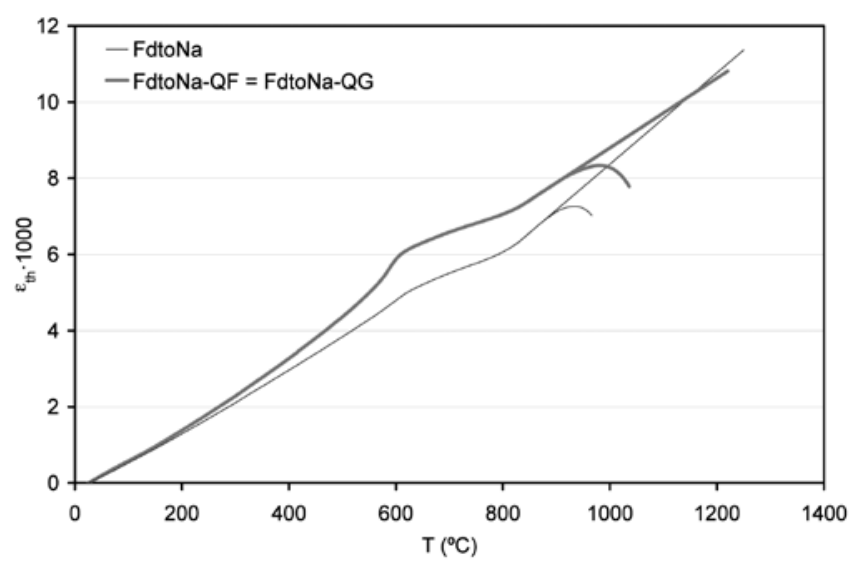

Figura 7. Curva dilatométrica extrapolada con un tramo lineal antes de la temperatura de reblandecimiento.
En la figura 9.a se muestra la evolución de las tensiones en la superficie de las piezas FdtoNa sometidas a diferentes tipos de enfriamientos. En el centro aparecen tensiones que tienen signo contrario a las superficiales, y que se no se han representado para simplificar la gráfica. En ningún caso por encima de la temperatura de transición vítrea $\left(\mathrm{T}_{\mathrm{g}}\right)$ aparecen tensiones. Esto se debe a la baja viscosidad del material, que relaja todas las tensiones en cuanto aparecen.

Por debajo de $\mathrm{T}_{g^{\prime}}$ en el enfriamiento ERR comienzan a aparecer las tensiones. A $573{ }^{\circ} \mathrm{C}$ aparece un pico asociado a la transición $\beta \rightarrow \alpha$ del cuarzo presente de forma residual en la muestra FdtoNa. Finalmente, a temperatura ambiente hay una tensión residual que no es nula.

Las piezas sometidas a enfriamiento ELL presentan un nivel de tensiones bajo en todo el intervalo, debido a que los gradientes térmicos son poco importantes. A temperatura ambiente aparece una tensión residual en torno a 1-2 MPa.

El enfriamiento ELR es idéntico al ELL hasta alcanzar los $650{ }^{\circ} \mathrm{C}$. Por debajo de esta temperatura las piezas se extraen del horno y comienza un enfriamiento rápido, surgiendo tensiones de tracción que alcanzan su máximo a la temperatura de transición del cuarzo. Superada esta temperatura, las tensiones se reducen alcanzándose valores similares a los del enfriamiento ELL.

A partir de esta gráfica se puede concluir que las tensiones residuales aparecen exclusivamente como consecuencia de los gradientes térmicos a elevada temperatura, ligeramente por encima de $\mathrm{T}_{g^{\prime}}$ como cabría esperar.

Las tensiones son función de la historia térmica; por ejemplo, si se comparan los enfriamientos ELR y ERR a 600

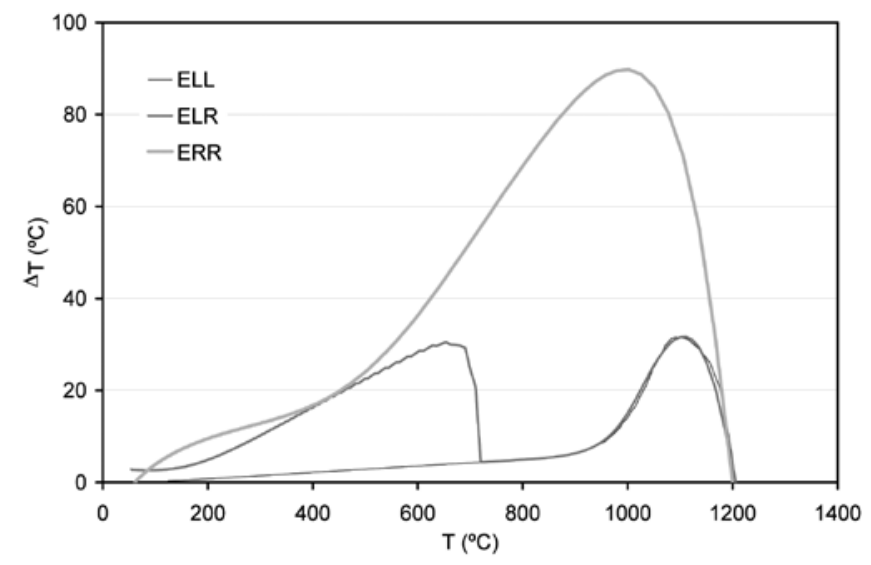

Figura 8. Diferencia térmica entre el centro y la superficie de las probetas FdtoNa some-tidas a los tres enfriamientos. 
${ }^{\circ} \mathrm{C}$ (figura 9.a) se constata que las tensiones son muy distintas, a pesar de que la diferencia entre la temperatura en superficie y el centro es parecida en ambos enfriamientos (figura 8). La causa de esto es que la deformación viscosa es muy diferente en ambas condiciones, debido a que la velocidad de enfriamiento es muy distinta a una temperatura por encima de $\mathrm{T}_{\mathrm{g}}$.

En la figura 9.b se presenta el efecto de la adición de cuarzo sobre las tensiones residuales en superficie para los enfriamientos ELR y ERR. La presencia de cuarzo induce una tensión de tracción en la superficie, lo que explica que las composiciones ricas en cuarzo sean más propensas a romperse durante el enfriamiento industrial.

Por otra parte, el nivel de tensiones generadas en torno a los $700{ }^{\circ} \mathrm{C}$ durante el enfriamiento ERR es menor cuando se añade cuarzo. Esto se debe a dos factores: la composición con cuarzo añadido presenta un menor coeficiente de dilatación térmico por encima de $700{ }^{\circ} \mathrm{C}$ (figura 7) y tiene una difusividad térmica mayor (figura 5). Una mayor difusividad térmica se traduce en menores diferencias térmicas entre la superficie y el centro.
La figura 10 muestra los perfiles de tensiones residuales experimentales y teóricos obtenidos para las probetas FdtoNa. Los correspondientes a las otras probetas son cualitativamente similares. La concordancia entre los valores teóricos y los experimentales es razonablemente buena, en especial teniendo en cuenta que el modelo no contiene ningún parámetro de ajuste.

Se constata que el perfil de tensiones es parabólico, de acuerdo a lo indicado por la ec. 4, estando la superficie sometida a compresión (tensión negativa) y el centro a tracción (tensión positiva). Esta distribución de tensiones refuerza la pieza, ya que la rotura en baldosas suele producirse por tensiones de tracción en la superficie. Las tensiones residuales son significativamente mayores en el enfriamiento ERR que en el ELR y ELL, en concordancia con los resultados de la figura 9.a.

El hecho de que el perfil sea parabólico implica que un único parámetro sirve para caracterizarlo; por ejemplo se puede utilizar la tensión residual en la superficie, como se indicó en la ec. 4. En la figura 11 se comparan los resultados teóricos

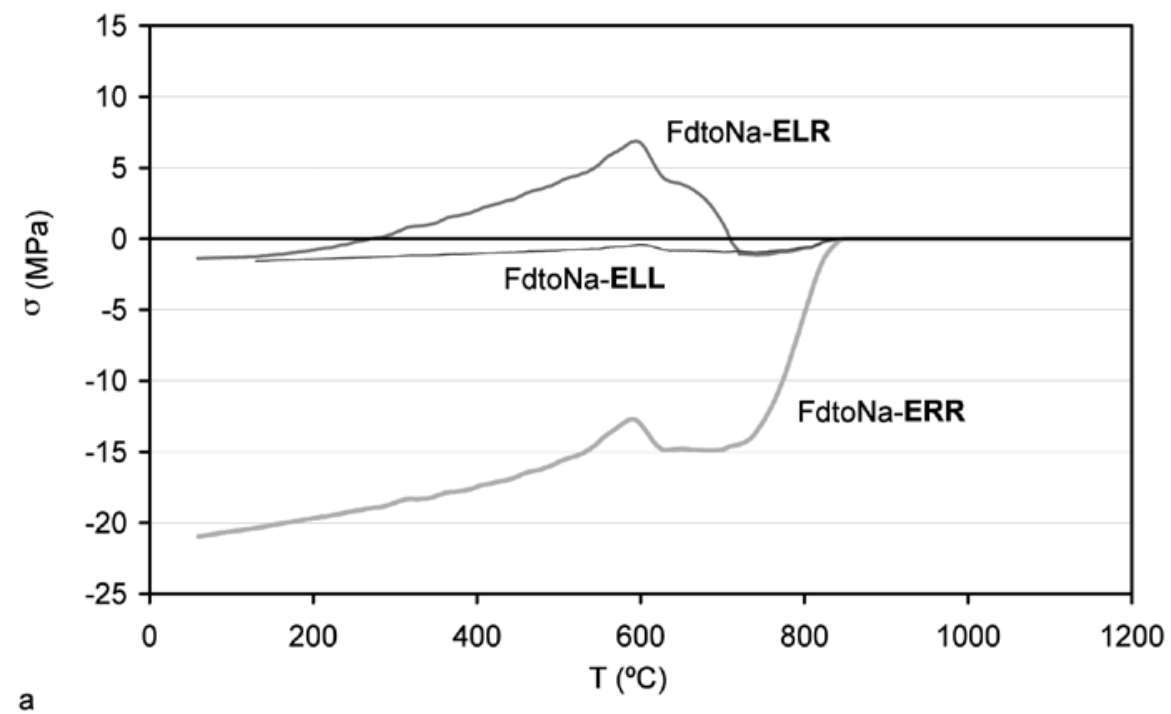

a

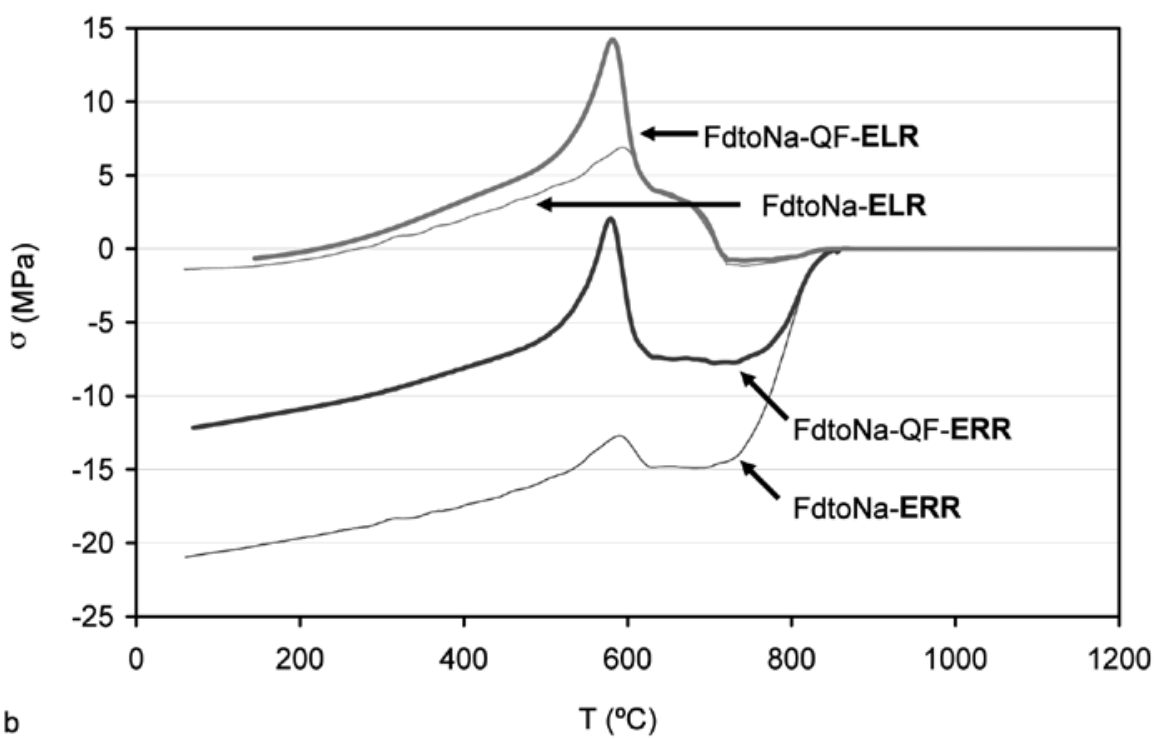

Figura 9. a) Evolución de la tensión en la superficie de las probetas FdtoNa sometidas a diferentes tipos de enfriamientos. b) Efecto del cuarzo sobre las tensiones residuales en superficie. 
y experimentales de las tensiones residuales en la superficie para las diferentes probetas y enfriamientos. Los resultados son razonablemente buenos; en particular, el modelo es capaz de predecir que las tensiones residuales en el enfriamiento ERR son menores para las probetas con cuarzo añadido, independientemente del tamaño de las partículas de cuarzo.

Otros factores no analizados, como el espesor, pueden influir también en las tensiones residuales. Cabría esperar que menores espesores llevaran a tensiones inferiores.

\section{CONCLUSIONES}

- Se ha desarrollado un modelo y una metodología de cálculo que permite estimar las tensiones residuales macroscópicas que se producen durante el enfriamiento. El modelo únicamente hace uso de datos de bibliografía y ensayos de laboratorio, pero no contiene ningún parámetro de ajuste.
- Los perfiles de tensiones son parabólicos. Esto permite utilizar un único parámetro, como es la tensión en la superficie, para caracterizarlos.

- Las tensiones residuales se generan únicamente a elevada temperatura. Velocidades de enfriamiento elevadas por debajo de $\mathrm{T}_{\mathrm{g}}$ (por ejemplo durante la transformación alotrópica del cuarzo) conducen a tensiones durante el enfriamiento, pero estas tensiones desaparecen a temperatura ambiente.

- Las tensiones durante el enfriamiento dependen de la historia térmica de la pieza. En particular, las tensiones en la zona de transición del cuarzo son mayores (más positivas o menos negativas) cuando el enfriamiento a alta temperatura se realiza de forma más lenta. Esto se explica por la deformación viscosa que se produce a elevada temperatura.

- La transformación alotrópica $\beta \rightarrow \alpha$ del cuarzo conduce a un pico en las tensiones durante el enfriamiento.

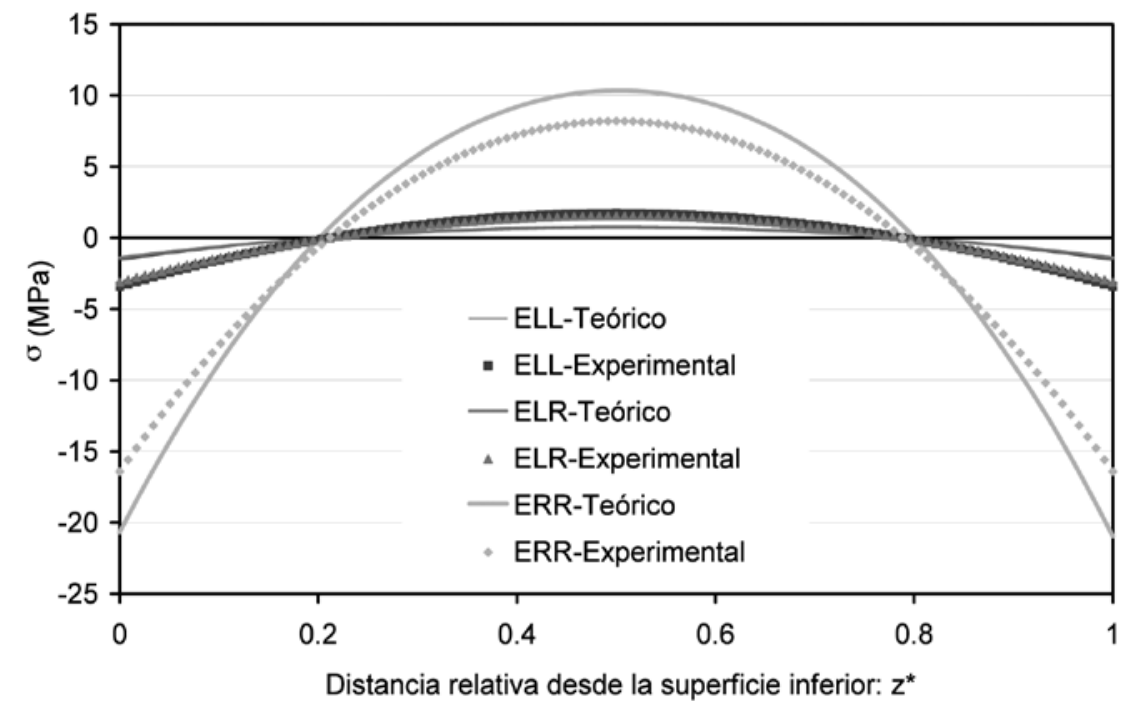

Figura 10.Perfiles de tensiones residuales experimentales y teóricas para las probetas FdtoNa.

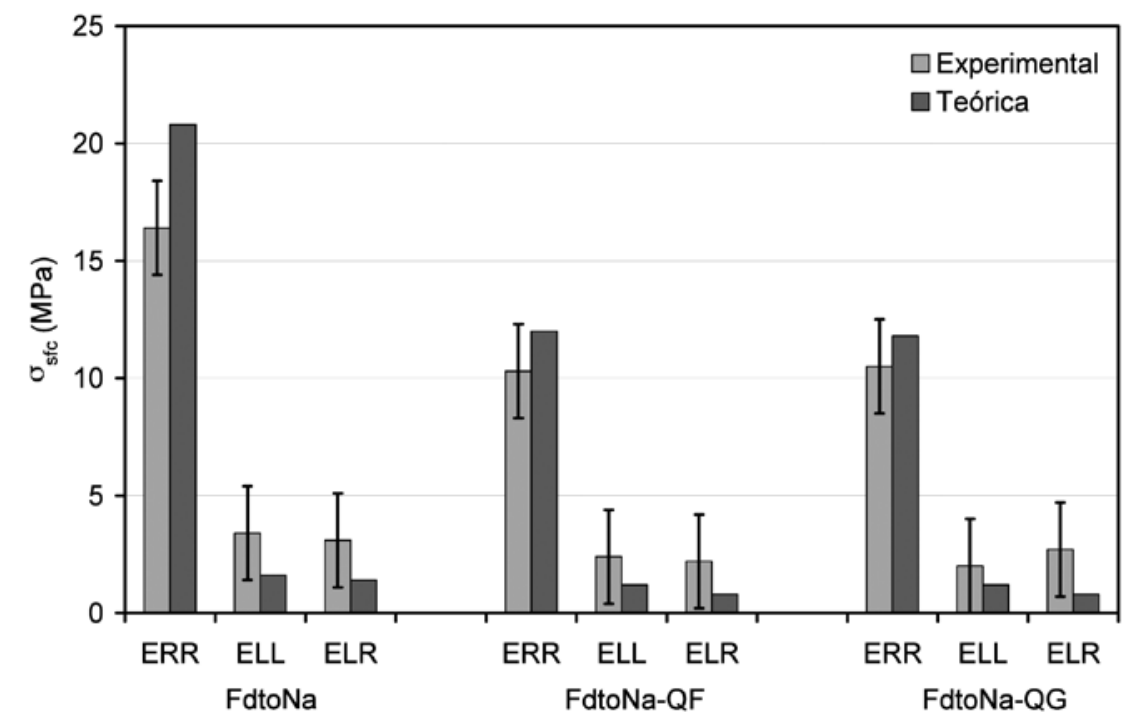

Figura 11. Tensiones residuales en superficie para las diferentes probetas y enfriamientos analizados. 
- $\mathrm{Al}$ incorporar cuarzo las tensiones residuales son menores, en las mismas condiciones de enfriamiento. Esto se debe a que a alta temperatura, al añadir cuarzo, se reduce el coeficiente de dilatación lineal y aumenta la difusividad térmica.

\section{AGRADECIMIENTOS}

Los autores desean expresar su agradecimiento al Ministerio de Ciencia e Innovación por la cofinanciación recibida por dentro del Programa Nacional de Proyectos de Investigación Fundamental (BIA2009-10692), y al proyecto CAPES-DGU (Brasil: BEX 6505/10-4).

\section{BIBLIOGRAFÍA}

(1) CANTAVELlA, V. et al. Evolución de las tensiones y curvaturas en soportes porosos durante el enfriamiento. En: X Congreso mundial de la calidad del azulejo y del pavimento cerámico - Qualicer 2008. Castellón: Cámara oficial de comercio, industria y navegación, 2008. pp. P.BC241-P.BC255.

(2) INCROPERA, F.P.; WITT, D.P. de. Fundamentals of heat and mass transfer. Singapore: John Wiley \& Sons, 1990.

(3) QUIROGA, A.S. Curso de elasticidad. Madrid: Bellisco, 1990.

(4) SCARFE, C. M.; CRONIN, D.J. Viscosity-temperature relationship of melts at $1 \mathrm{~atm}$ in the system diopside-albite. Am. Mineral., 71(5-6), 767-771, 1986.

(5) NONI, A DE JR.; HOTZA, D.; CANTAVELLA, V.; SÁNCHEZ, E. Influence of macro-scopic residual stresses on the mechanical behavior and microstructure of porcelain tile. J. Eur. Ceram. Soc., 28 (13), 2463-2469, 2008

(6) LU, J. Handbook of Measurement of Residual Stresses. Fairmont Press, Lilburn, 1996.

(7) CANTAVELlA, V.; GARCÍA-TEN, J.; SÁNCHEZ, E.; BANNIER, E.; SÁNCHEZ, J.; SOLER, C.; SALES, J. Curvaturas diferidas en gres porcelánico. Análisis y medida de los factores que intervienen. En: $X$ Congreso mundial de la calidad del azulejo y del pavimento cerámico - Qualicer 2008. Castellón: Cámara oficial de comercio, industria y navegación, 2008. pp. P.BC207-P.BC224.
(8) DAVIS, H.T.; VALENCOURT, L.R.; JOHNSON, C.E. Transport processes in composite media. J. Am. Ceram. Soc., 58(9-10), 446-452, 1975.

(9) WANG, M. ; PAN, N. Predictions of effective physical properties of complex multi-phase materials. Mater. Sci. Eng., R, 63(1), 1-30, 2008.

(10) CARSON, J.K.; LOVATT, S.J.; TANNER, D.J. et al. Thermal conductivity bounds for isotropic, porous materials. Int. J. Heat Mass Transfer, 48(11), 2150-2158, 2005.

(11) SCHNEIDER, S.J. Engineering properties of glass-ceramics, Engineered Materials Handbook, Vol. 4: Ceramics and Glasses, ASM International, USA (1991).

(12) HOFMEISTER, A.M.; WHITTINGTON, A.G.;PERTERMANN, M Transport properties of high albite crystals, near-endmember feldspar and pyroxene glasses, and their melts to high temperature. Contrib. Mineral Petrol, 158(3), 381-400, 2009.

(13) GIBERT, B. ; MAINPRICE, D. Effect of crystal preferred orientation on the thermal diffusivity of quartz polycrystalline aggregates at high temperature. Tectonophysics, 465(1-4), 150-163, 2009.

(14) WEAST, R.C. Handbook of chemistry and physics. CRC Press, Cleveland, OH, 1974.

(15) OHNO, I.; HARADA, K.; YOSHITOMI, C. Temperature variation of elastic constants of quartz across the $\alpha-\beta$ transition. Phys Chem Minerals, 33(1), $1-9,2006$.

(16) HEMINGWAY, B.S. Quartz: Heat Capacities from 340 to $1000 \mathrm{~K}$ and revised values for thermodynamic properties. Am. Mineral., 72, 273-279, 1987.

(17) AHRENS, I. Mineral Physics and Crystallography: A Handbook of Physical Constants. American Geophysical Union, 1995.

(18) ROUXEL, T. Elastic Properties and Short-to Medium-Range Order in Glasses. J. Am. Ceram. Soc., 90(10), 3019-3039, 2007.

(19) MAGRINI, F.; FERRARI, R.; BRUNETTI, P. Ruolo della viscositá e della tensione superficiale nel processo di cottura delle vetrine e degli smalti ceramici. Cerâmica Informazione, 171, 391, 1979.

(20) NEVES, E; POFFO, E.D ; FREDEL, M.C. RIELLA, H.G; ALARCON, O.E. Efeito da adição de $\mathrm{Na}_{2} \mathrm{O}$ na viscosidade e devitrificação do vidro obtido a partir de cinzas volantes de $\mathrm{Li}_{2} \mathrm{O}$. Química Nova, 21(4), 534-537, 1998.

Recibido: 01/03/2012

Aceptado: 11/04/2012 